\title{
Constructing projective varieties in weighted flag varieties
}

\author{
Muhammad Imran Qureshi and Balázs Szendrői
}

\begin{abstract}
We compute the Hilbert series of general weighted flag varieties and discuss a computer-aided method to determine their defining equations. We apply our results to weighted flag varieties coming from the Lie groups of type $G_{2}$ and GL(6), to construct some families of polarised projective varieties in codimensions 8 and 6 , respectively.
\end{abstract}

\section{Introduction}

The aim of this article is twofold. Firstly, we prove a formula for the Hilbert series of general weighted flag varieties of Grojnowski and Corti-Reid [6] and discuss a computer-aided method to determine their defining equations. The Hilbert series is expressed in terms of Lie-theoretic data associated to the weighted flag variety. The equations are determined using an explicit construction of highest weight modules over Lie algebras. Secondly, we use these results to construct families of projective varieties in higher codimension by taking quasi-linear sections of weighted flag varieties. As examples, we exhibit in this article some new families of Calabi-Yau threefolds in codimensions 6 and 8. A more complete list of families arising from this construction will be given elsewhere [11.

We are interested in polarized varieties $(X, D)$, projective varieties $X$ polarised by a $\mathbb{Q}$-ample Weil divisor $D$ such that some integer multiple of $D$ is Cartier. Such a polarized variety $(X, D)$ gives rise to a finitely generated graded ring

$$
R(X, D)=\bigoplus_{n \geq 0} H^{0}(X, n D)
$$

A surjection

$$
k\left[x_{0}, \cdots, x_{n}\right] \rightarrow R(X, D)
$$

from a free graded ring $k\left[x_{0}, \ldots, x_{n}\right]$ generated by variables $x_{i}$ of weights $w_{i}$ gives an embedding of $(X, D)$ into weighted projective space

$$
i: X=\operatorname{Proj} R(X, D) \hookrightarrow \mathbb{P}\left[w_{0}, \cdots, w_{n}\right]
$$

with the divisorial sheaf $\mathcal{O}_{X}(D)$ being isomorphic to $\mathcal{O}_{X}(1)=i^{*} \mathcal{O}_{\mathbb{P}}(1)$. 
We are looking for examples where this embedding is of relatively low codimension. We work in the framework reviewed in [2], where the classical case of codimension at most 3 is discussed. See [1] for codimension 4 examples, and Corti and Reid [6] for examples in codimension 5 .

We construct examples in codimensions 6 and 8 arising as quasilinear sections of particular weighted flag varieties $\left(w \Sigma, \mathcal{O}_{w \Sigma}(1)\right)$ embedded in weighted projective space by their natural Plücker-type embeddings. We look for candidate examples by computing the Hilbert series of a weighted flag variety of a given type. To understand the singularities of quasilinear sections, we need to know the defining equations of the flag variety. Defining ideals of flag varieties are described in [9, Sec 1] in Lie-theoretic terms; we work out the equations by a GAP4 code, using an explicit construction of representations which appears in [8]. In theory, this approach allows one to search for examples in arbitrarily high fixed codimension.

After fixing notation in Section 2, in Section 3 we prove a formula for the Hilbert series $P_{w \Sigma}$ of a weighted flag variety $w \Sigma$. We also describe a method of determining the defining equations, and explain how to construct families of polarized varieties such as Calabi-Yau threefolds as quasi-linear sections of $w \Sigma$. In Section 4, we study weighted flag varieties associated to the Lie group of type $G_{2}$, leading to the codimension eight varieties. The case of weighted homogeneous $w \mathrm{Gr}(2,6)$ in codimension six is discussed in Section 5. Complete lists of families that can be constructed in these ambient varieties, as well as other examples of higher codimension, will appear elsewhere [11].

\section{Acknowledgements}

We wish to thank Willem De Graaf for providing us with the GAP4 code to compute the defining equations of flag varieties and Richard Williamson for further help with GAP. We also thank Gavin Brown and Miles Reid for helpful discussions. The first author has been supported by a grant from the Higher Education Commission (HEC) of Pakistan.

\section{Definitions and conventions}

\subsection{Algebraic geometry}

We work over a field $\mathbb{C}$ of complex numbers. A polarised variety is a pair $(X, D)$, where $X$ is a normal projective algebraic variety and $D$ is an ample $\mathbb{Q}$-Cartier Weil divisor on $\mathrm{X}$.

For positive weights $w_{0}, w_{1}, \cdots, w_{n}$, we use the standard notation $\mathbb{P}\left[w_{0}, w_{1}, \cdots, w_{n}\right]$ for weighted projective space; sometimes we will write $w \mathbb{P}$ if the weights are clear. The weighted projective space $\mathbb{P}\left[w_{i}\right]$ is called well-formed, if no $n-1$ of $w_{0}, \cdots, w_{n}$ have a common factor. The point $P_{i} \in w \mathbb{P}$ with coordinates $[0, \ldots, 0,1,0, \ldots, 0]$, where the 1 is in the $i$-th position, is a vertex, the 1-dimensional stratum $P_{i} P_{j}$ an edge, etc. Defining

$h_{i, j, \ldots}=\operatorname{hcf}\left(w_{i}, w_{j}, \ldots\right)$, the vertex $P_{i}$ of a well-formed weighted projective space is a 
singularity of type

$$
\frac{1}{w_{i}}\left(w_{0}, \ldots, \widehat{w}_{i}, \ldots, w_{n}\right) .
$$

Each generic point $P$ of the edge $P_{i} P_{j}$ has an analytic neighbourhood $P \in U$ which is analytically isomorphic to $(0, Q) \in \mathbb{A}^{1} \times X$, where $Q \in X$ is a singularity of type

$$
\frac{1}{h_{i, j}}\left(w_{0}, \ldots, \widehat{w}_{i}, \ldots, \widehat{w}_{j}, \ldots, w_{n}\right) \text {. }
$$

Similar results hold for higher dimensional strata.

A projective subvariety $X \subset \mathbb{P}^{n}\left[w_{i}\right]$ of codimension $c$ is called well-formed, if $\mathbb{P}^{n}\left[w_{i}\right]$ is well-formed and $X$ does not contain a codimension $c+1$ singular stratum of $\mathbb{P}\left[w_{i}\right]$. The subvariety $X \subset \mathbb{P}\left[w_{i}\right]$ is quasi-smooth, if the affine cone $\widetilde{X} \subset \mathbb{A}^{n+1}$ of $X$ is smooth outside its vertex $\underline{0}$. If $X$ is quasi-smooth, then it will only have quotient singularities induced by the singularities of $\mathbb{P}\left[w_{i}\right]$. The subvariety $X \subset \mathbb{P}\left[w_{i}\right]$ will always be assumed to be polarized by the restriction of the tautological ample divisor $\mathcal{O}_{\mathbb{P}}(1)$.

The Hilbert series of a polarised projective variety $(X, D)$ is

$$
P_{(X, D)}(t)=\sum_{n \geq 0} \operatorname{dim} H^{0}(X, n D) t^{n}
$$

We will sometimes write $P_{X}(t)$ if no confusion can arise. Appropriate Riemann-Roch formulas, together with vanishing, can be used to compute $h^{0}(X, n D)=\operatorname{dim} H^{0}(X, n D)$ in favourable cases.

A polarised Calabi-Yau threefold $(X, D)$ is a Gorenstein, normal, projective three dimensional algebraic variety with $K_{X} \sim 0$ and $H^{1}\left(X, \mathcal{O}_{X}\right)=H^{2}\left(X, \mathcal{O}_{X}\right)=0$. We allow $(X, D)$ to have at worst canonical quotient singularities, consisting of points and curves on $X$. If $C$ is a curve of singularities of such an $(X, D)$, take a generic surface $S \subset X$ such that $C$ and $S$ intersect transversely in finite number of points. If every point in the intersection is a singular point of type $\frac{1}{r}(1,-1)$ on polarised surface $\left(S,\left.D\right|_{S}\right)$, then $C \in X$ is called a curve of singularities of type $\frac{1}{r}(1,-1)$, known as an $A_{r-1}$ curve. Each $A_{r-1}$ curve contains a finite number of dissident points, where the singularity is worse. If an $A_{r-1}$ curve $C$ contains the dissident points $\left\{P_{\eta}: \eta \in \Lambda\right\}$ of type $\frac{1}{r \tau_{\eta}}\left(w_{1 \eta}, w_{2 \eta}, w_{3 \eta}\right)$, then we define the index of $C$ to be

$$
\tau_{C}=\operatorname{lcm}_{\eta \in \lambda}\left\{\tau_{\eta}\right\} .
$$

For an $A_{r-1}$ curve $C$ with no dissident points, $\tau_{C}=1$. The contribution of $C$ to the Riemann-Roch formula [5] is determined by $\tau_{C}$ as well as a further invariant $N_{C}$ depending on the normal bundle of $C$ in $X$; see [5, p.16] for a detailed description.

\subsection{Representation theory}

Let $G$ to be a reductive Lie group with corresponding Lie algebra $\mathfrak{g}$. We denote by $B$ a Borel subgroup of $G$, by $P$ a parabolic subgroup of $G$, and by $T$ a maximal torus, such that $T \subset B \subset P \subset G$. Let $\Lambda_{W}=\operatorname{Hom}\left(T, \mathbb{C}^{*}\right)$ be the weight lattice. 
We recall that any irreducible representation $V$ of $G$ has a decomposition

$$
V=\bigoplus_{\alpha \in \Lambda_{W}} V_{\alpha}
$$

into eigenspaces under the action of the maximal torus $T$; the $\alpha$ 's with nonzero $V_{\alpha}$ are the weights of the representation $V$. The nonzero weights of the adjoint representation are called the roots. The root lattice is the sublattice $\Lambda_{R}$ of $\Lambda_{W}$ generated by the roots. Their dual lattices of one-parameter subgroups are denoted by $\Lambda_{R}^{*}$ and $\Lambda_{W}^{*}$ respectively; we have a perfect pairing $<,>: \Lambda_{W} \times \Lambda_{W}^{*} \rightarrow \mathbb{Z}$.

We denote the set of roots by $\nabla$ and fix a decomposition into positive and negative roots, $\nabla_{+} \cup \nabla_{-}$. The set of positive roots that can not be written as a sum of two other positive roots form the set of simple roots $\nabla_{0}$ of $G$. Any root $\alpha \in \nabla$ determines an involution of the maximal torus $\mathrm{T}$, and hence a reflection $s_{\alpha}$. These reflections generate the Weyl group $W$ of $G$. The reflections corresponding to the simple roots are called simple reflections.

There is a partial order defined on the weights of any representation by $\alpha \leq \beta$ if and only if $\beta-\alpha$ is non-negative linear combination of simple roots. A weight $\lambda$ of a representation is called the highest or dominant weight of the representation if no other weight is greater than $\lambda$ with respect to this partial order. For semisimple $G$, there is a set of weights $\omega_{1}, \cdots, \omega_{n}$ in $\Lambda_{W}$, called the fundamental weights, defined by the property that any dominant weight can be written uniquely as a non-negative integral linear combination of the fundamental weights.

The quotient of the Lie group $G$ by a Borel subgroup, $\Sigma=G / B$ is the complete flag variety. Quotients of the form $\Sigma=G / P$, for $P \subset G$ a parabolic, are called (generalized) flag varieties or projective homogeneous spaces in the literature; we will use the words flag variety and homogeneous variety interchangeably.

Let $p \in \mathbb{P} V_{\lambda}$ be the highest weight vector in the representation $V_{\lambda}$ of $G$ with highest weight $\lambda \in \Lambda_{W}$. Let $P_{\lambda}$ be the parabolic subgroup corresponding to those elements in $\nabla_{0}$, which are orthogonal to the weight $\lambda$ in the weight lattice. Then the unique closed orbit $G$.p of the action of $G$ on $\mathbb{P} V_{\lambda}$ realizes the flag variety $\Sigma=G / P_{\lambda}$ as a projective subvariety of $\mathbb{P} V_{\lambda}$. All flag varieties $G / P$ can be realised as projective varieties in this way.

As an example, recall that if $G=\operatorname{SL}(n, \mathbb{C})$, then $B$ is a subgroup of upper triangular matrices, and $G / B$ is the variety of all flags

$$
\Sigma=\operatorname{SL}(n, \mathbb{C}) / B=\left\{0 \subset V_{1} \subset V_{2} \subset \cdots \subset V_{n}=V\right\}
$$

of subspaces, with $\operatorname{dim} V_{k}=k$. The group $G$ acts transitively on $\Sigma$ and $B$ is the stabilizer of a fixed flag. If $P$ is a parabolic subgroup of $S L(n, \mathbb{C})$ containing $B$ then the quotient

$$
\Sigma=\operatorname{SL}(n, \mathbb{C}) / P=\left\{0 \subset V_{i} \subset V_{m} \subset \cdots \subset V_{n}\right\}
$$

is a (generalised) flag variety, with $P$ being the stabilizer of a fixed partial flag. 


\section{$3 \quad$ Weighted flag varieties}

\subsection{Hilbert series formula}

We start by recalling the notion of weighted flag variety due to Grojnowski and Corti-Reid [6]. Fix a reductive Lie group $G$ as above, and a highest weight $\lambda \in \Lambda_{W}$. Let $\Sigma=G / P_{\lambda}$ be the corresponding flag variety as above. Choose $\mu \in \Lambda_{W}^{*}$ and an integer $u \in \mathbb{Z}$ such that

$$
<w \lambda, \mu>+u>0
$$

for all elements $w$ of the Weyl group. Consider the affine cone $\widetilde{\Sigma} \subset \widetilde{V_{\lambda}}$ of the embedding $\Sigma \hookrightarrow \mathbb{P} V_{\lambda}$, and divide it by the $\mathbb{C}^{*}$-action on $V_{\lambda}-\{0\}$ given by

$$
\left(\varepsilon \in \mathbb{C}^{*}\right) \mapsto\left(v \mapsto \varepsilon^{u}(\mu(\varepsilon) \circ v)\right)
$$

Denote this variety by $w \Sigma(\mu, u)$ or $w \Sigma$ if no confusion can arise. The inequality (3.1) ensures that all the weights on $w \Sigma(\mu, u) \subset w \mathbb{P} V_{\lambda}$ are positive, so the quotient indeed exists.

3.2 Theorem The Hilbert series of the weighted flag variety $(w \Sigma(\mu, u), D)$ has the following closed form.

$$
P_{w \Sigma}(t)=\frac{\sum_{w \in W}(-1)^{w} \frac{t^{<w \rho, \mu>}}{\left(1-t^{<w \lambda, \mu>+u}\right)}}{\sum_{w \in W}(-1)^{w} t^{<w \rho, \mu>}} .
$$

Here $\rho$ is the Weyl vector, half the sum of the positive roots of $G$, and $(-1)^{w}=1$ or -1 depending on whether $w$ consists of an even or odd number of simple reflections in the Weyl group $W$.

Proof Any weight $\lambda$ of $G$-representation $V_{\lambda}$ gives rise to a line bundle $\mathcal{L}_{\lambda}$ on the straight flag variety $\Sigma$. If $\lambda$ is dominant, then by the Borel-Bott-Weil theorem, the space of holomorphic sections of the line bundle $\mathcal{L}_{\lambda}$ is the irreducible representation with highest weight $\lambda$.

Let $D=\mathcal{O}_{w \Sigma}(1)$ under the embedding $w \Sigma \subset w \mathbb{P} V_{\lambda}$, then we have a graded ring

$$
R=\bigoplus_{n \geq 0} H^{0}\left(w \Sigma, \mathcal{O}_{w \Sigma}(n D)\right)
$$

By construction, $\operatorname{Spec}(R)=\widetilde{w \Sigma}$, the affine cone over the weighted flag variety $w \Sigma$. However, this affine cone is the same as the straight affine cone $\widetilde{\Sigma}$, so by the Borel-Bott-Weil theorem, we have

$$
R=\bigoplus_{m \geq 0} V_{m \lambda}
$$


The grading on $R$ given by (3.3) is different from the grading given by (3.4) because of the weights. The Hilbert series of the graded ring (3.3) is given by

$$
P_{w \Sigma}(t)=\sum_{n \geq 0} h^{0}\left(w \Sigma, \mathcal{O}_{w \Sigma}(n D)\right) t^{n}
$$

By the Weyl character formula,

$$
\operatorname{Char}\left(V_{\lambda}\right)=\frac{\sum_{w \in W}(-1)^{w} t^{w(\lambda+\rho)}}{\sum_{w \in W}(-1)^{w} t^{w(\rho)}} .
$$

Therefore by (3.5) and (3.6), the Hilbert series of the weighted flag variety with weights $\langle w \lambda, \mu\rangle+u$, which are positive by (3.1), is given by the infinite sum

$$
P_{w \Sigma}(t)=\sum_{n \geq 0} \frac{\sum_{w \in W}(-1)^{w} t^{<w(n \lambda+\rho), \mu>+n u}}{\sum_{w \in W}(-1)^{w} t<w \rho, \mu>} .
$$

After rearrangement of terms we get the claimed formula (3.2).

3.3 Remark Since (3.2) involves summing over the Weyl group, it is best to use a computer algebra system for explicit computations. We used GAP, SAGE and Mathematica in combination to work out the Hilbert series formulas for the flag varieties appearing below. As immediate examples, note that the formulas appearing in [6, p.8 and p.23] can be derived from (3.2) with little effort.

3.4 Remark Note that by the Weyl denominator identity, the denominator of our expression for the Hilbert series has two equivalent forms

$$
\sum_{w \in W}(-1)^{w} t^{<w \rho, \mu>}=t^{<\rho, \mu>} \prod_{\alpha \in \nabla_{+}}\left(1-t^{<-\alpha, \mu>}\right) .
$$

Note also that by standard Hilbert-Serre theorem [3, Theorem 11.1], the Hilbert series of the weighted flag variety has a reduced expression

$$
P_{w \Sigma}(t)=\frac{N(t)}{\prod_{w_{i} \in \Lambda_{W}}\left(1-t^{<w_{i}, \mu>+u}\right)} .
$$

Here the product is over all the weights of the representation $V_{\lambda}$; the polynomial $N(t)$ is called the Hilbert numerator of the Hilbert series. There is a lot of cancellation happening when going from (3.2) to (3.8).

\subsection{Equations of flag varieties}

The flag variety $\Sigma=G / P \hookrightarrow \mathbb{P} V_{\lambda}$ is defined by an ideal $I=<Q>$ of quadratic equations generating a linear subspace $Q \subset Z=S^{2} V_{\lambda}^{*}$ of the second symmetric power of the contragradient representation $V_{\lambda}^{*}$. The $G$-representation $Z$ has a decomposition

$$
Z=V_{2 \nu} \oplus V_{1} \oplus \cdots \oplus V_{n}
$$


into irreducible direct summands, with $\nu$ being the highest weight of the representation $V_{\lambda}^{*}$. As discussed in [9, 2.1], the subspace $Q$ in fact consists of all the summands except $V_{2 \nu}$.

We compute these equations by using GAP4 code which follows an algorithm given in [8]. The ideal $I$ is constructed as a certain left ideal of the Verma module corresponding to the highest weight $\lambda$ of the Lie algebra $\mathfrak{g}$. In practice, fix a Lie algebra $\mathfrak{g}$ and dominant weight $\lambda$. Take the left action of $\mathfrak{g}$ on its universal enveloping algebra $U(\mathfrak{g})$ and construct the Verma module $M(\lambda)=U(\mathfrak{g}) / N(\lambda)$, where $N(\lambda)$ is a certain $\mathfrak{g}$-submodule of $U(\mathfrak{g})$. The module $M(\lambda)$ is also an algebra, and there exist a certain left ideal $I(\lambda)$ of it such that $M(\lambda) / I(\lambda)$ is an irreducible module of highest weight $\lambda$. One can construct the last quotient by calculating a Gröbner basis of $I(\lambda)$.

The defining equations of the flag varieties appearing in [6, p.4 and p.20] can easily be recovered using this algorithm, implemented in the GAP4 [12] code given in the Appendix.

\subsection{Constructing polarized varieties}

We recall the different steps in the construction of polarized varieties as quasi-linear sections of weighted flag varieties. We will concentrate on the case of Calabi-Yau threefolds; obvious modifications apply for different classes of varieties (Fano, general type, etc).

1. Choose embedding. We choose a reductive Lie group $G$ and a $G$-representation $V_{\lambda}$ of dimension $n$ with highest weight $\lambda$. We get a straight flag variety $\Sigma=G / P_{\lambda} \hookrightarrow$ $\mathbb{P} V_{\lambda}$ of computable dimension $d$ and codimension $c=n-1-d$. We choose $\mu \in \Lambda_{W}^{*}$ and $u \in \mathbb{Z}$ to get an embedding $w \Sigma \hookrightarrow w \mathbb{P} V_{\lambda}\left[w_{i}\right]$. The equations of this embedding can be found as described above.

2. Compute Hilbert series and $K_{w \Sigma}$. We compute the Hilbert series of $w \Sigma$ by expanding and simplifying (3.2) for the given values of $\lambda, \mu, u$. If $w \Sigma$ is well-formed, we can derive the canonical divisor class $K_{w \Sigma}$ of $w \Sigma$.

3. Take threefold Calabi-Yau section of $w \Sigma$. We take a quasi-linear complete intersection

$$
X=w \Sigma \cap\left(w_{1}\right) \cap \cdots \cap\left(w_{k}\right)
$$

inside $w \Sigma$ of generic hypersurfaces of degrees equal to some of the weights $w_{i}$. We choose values so that $K_{X}=0$ and $\operatorname{dim}(X)=d-k=3$. This gives the embedding $X \hookrightarrow \mathbb{P}\left[w_{0}, \cdots, w_{s}\right]$, with $s=n-k$. More generally, as in [6], we can take complete intersections inside projective cones over $w \Sigma$, adding weight one variables to the coordinate ring which are not involved in any relation.

4. Check singularities. As we are interested in quasi-smooth Calabi-Yau threefolds, all the singularities of $X$ must be induced by the weights of $\mathbb{P}^{s}\left[w_{i}\right]$. Singular strata of $\mathbb{P}^{s}\left[w_{i}\right]$ correspond to sets of weights $w_{i_{0}}, \cdots, w_{i_{p}}$ with $\operatorname{gcd}\left(w_{i_{0}}, \cdots, w_{i_{p}}\right)=r$ nontrivial, defining a singular stratum $S \subset w \mathbb{P}$. If the intersection $Y=X \cap S$ is non-empty, it has to be a point or a curve, and we need to find local coordinates in neighbourhoods of points $P \in Y$ to check the local transversal structure. 


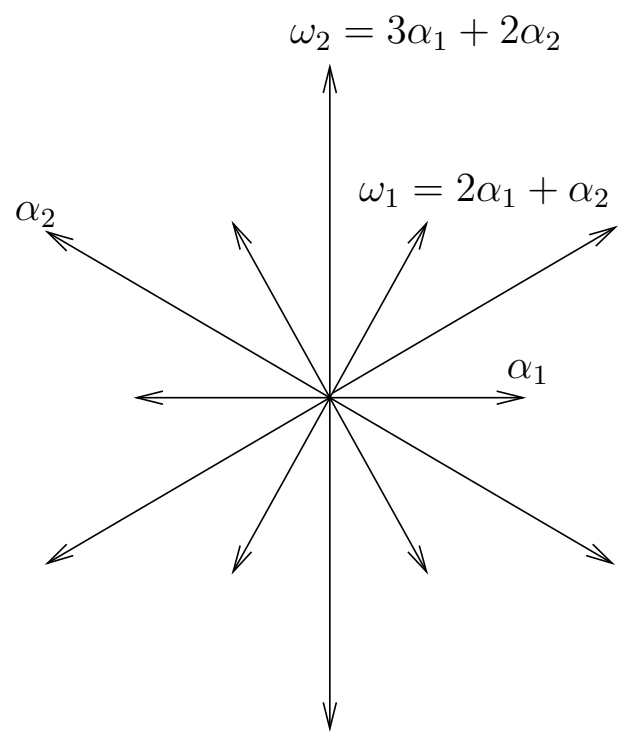

Figure 1: Root System of $G_{2}$

5. Find invariants and check consistency. Using the orbifold Riemann-Roch formula of [5, Section 3], we can compute the invariants of the singular points in our family from the first few values of $h^{0}(n D)$, and verify that the same Hilbert series can be recovered.

\section{Weighted $G_{2}$ flag varieties}

\subsection{Generalities}

Consider the simple Lie group of type $G_{2}$. Denote by $\alpha_{1}, \alpha_{2} \in \Lambda_{W}$ a pair of simple roots of the root system $\nabla$ of $G_{2}$, taking $\alpha_{1}$ to be the short simple root and $\alpha_{2}$ the long one; see Figure 1. The fundamental weights are $\omega_{1}=2 \alpha_{1}+\alpha_{2}$ and $\omega_{2}=3 \alpha_{1}+2 \alpha_{2}$. The sum of the fundamental weights, which is equal to half the sum of the positive roots, is $\rho=5 \alpha_{1}+3 \alpha_{2}$. We partition the set of roots into long and short roots as $\nabla=\nabla_{l} \cup \nabla_{s} \subset \Lambda_{W}$. Let $\left\{\beta_{1}, \beta_{2}\right\}$ be the basis of the lattice $\Lambda_{W}^{*}$ dual to $\left\{\alpha_{1}, \alpha_{2}\right\}$.

\subsection{The $G_{2}$ hypersurface}

As a warmup exercise, consider the highest weight $\lambda_{1}=\omega_{1}=2 \alpha_{1}+\alpha_{2}$. The irreducible $G_{2^{-}}$ representation $V_{\lambda_{1}}$ is seven-dimensional [7, Chapter 22], and its projectivisation contains the five-dimensional flag variety $\Sigma \hookrightarrow \mathbb{P}^{6}$ as a hypersurface. The weighted version is obtained by letting $\mu=a \beta_{1}+b \beta_{2} \in \Lambda_{W}^{*}$ and $u \in \mathbb{Z}$, leading to the weighted homogeneous variety

$$
w \Sigma=w \Sigma(\mu, u) \subset w \mathbb{P} V_{\lambda_{1}} .
$$


4.3 Proposition The Hilbert series of weighted $G_{2}$ flag variety embedded in the $G_{2^{-}}$ representation with highest weight $\omega_{1}=\lambda_{1}=2 \alpha_{1}+\alpha_{2}$ is

$$
P_{w \Sigma}(t)=\frac{\left(1-t^{2 u}\right)}{\left(1-t^{u}\right) \prod_{\alpha \in \nabla_{s}}\left(1-t^{<\alpha, \mu>+u}\right)} .
$$

Moreover, if $w \Sigma$ is well-formed, then $K_{w \Sigma}=\mathcal{O}(-5 u)$.

Proof The set of weights on $w \Sigma \subset w \mathbb{P} V_{\lambda_{1}}$ is given by $\left\langle w \lambda_{1}, \mu\right\rangle+u$ for elements $w$ of the Weyl group. Since $\lambda_{1}$ is a short root, the images of $\lambda_{1}$ under the action of the Weyl group $W$ are exactly all the short roots appearing in Figure 1. We expand the formula (3.2) for $\lambda_{1}=\omega_{1}$ and $W=D_{12}$, the dihedral group on 6 letters, and we get

$$
P_{w \Sigma}(t)=\frac{\left(1+t^{u}\right)}{\prod_{\alpha \in \nabla_{s}}\left(1-t^{<\alpha, \mu>+u}\right)} .
$$

The set of weights on $w \mathbb{P} V_{\lambda_{1}}$ is

$$
\left\{u,<\alpha, \mu>+u \text { for all roots } \alpha \in \nabla_{s}\right\} .
$$

We multiply and divide by $\left(1-t^{u}\right)$ to get the full expression for the Hilbert series, so our denominator contains all weights on $w \mathbb{P} V_{\lambda_{1}}$. This gives the Hilbert series in the form of (4.1) which is in the general form (3.8). The sum of the weights on $w \mathbb{P} V_{\lambda_{1}}$ is $7 u$, therefore $K_{w \Sigma}=\mathcal{O}(-5 u)$.

Thus, as is well known, the straight homogeneous variety with $\mu=(a, b)=(0,0)$ and $u=1$ is a quadric hypersurface in $\mathbb{P}^{6}$ :

$$
P_{\Sigma}(t)=\frac{1-t^{2}}{(1-t)^{7}}
$$

\subsection{The codimension eight weighted $G_{2}$-variety}

We consider the $G_{2}$-representation with highest weight $\lambda_{2}=\omega_{2}=3 \alpha_{1}+2 \alpha_{2}$. The dimension of $V_{\lambda_{2}}$ is 14 [7, Chapter 22]. The homogeneous variety $\Sigma \subset \mathbb{P} V_{\lambda_{2}}$ is five dimensional, so we have an embedding $\Sigma^{5} \hookrightarrow \mathbb{P}^{13}$ of codimension 8. To work out the weighted version in this case, take $\mu=a \beta_{1}+b \beta_{2} \in \Lambda_{W}^{*}$ and $u \in \mathbb{Z}$.

4.5 Proposition The Hilbert series of the codimension eight weighted $G_{2}$ flag variety is given by

$$
P_{w \Sigma}(t)=\frac{1-\left(4+2 \sum_{\alpha \in \nabla_{s}} t^{<\alpha, \mu>}+\sum_{\alpha \in \nabla_{s}} t^{2<\alpha, \mu>}+\sum_{\alpha \in \nabla_{l}} t^{<\alpha, \mu>}\right) t^{2 u}+\cdots+t^{11 u}}{\left(1-t^{u}\right)^{2} \prod_{\alpha \in \nabla}\left(1-t^{<\alpha, \mu>+u}\right)} .
$$

Moreover, if $w \Sigma$ is well-formed, then the canonical bundle is $K_{w \Sigma}=\mathcal{O}(-3 u)$. 
Proof The set of weights on $G_{2}$-representation with highest weight $\lambda_{2}=\omega_{2}$ consists of all the roots given in Figure 1. The zero weight space appears with multiplicity two. Therefore the set of weights on $w \mathbb{P} V_{\lambda_{2}}$ is given by

$$
\left\{u, u,<\alpha, \mu>+u \text { for all } \alpha \in \nabla \text { of } G_{2}\right\} \text {. }
$$

We compute the Hilbert series expression (3.2) for $\lambda_{2}=\omega_{2}$ and Weyl group $W=D_{12}$. We get the following form of the Hilbert series.

$$
P_{w \Sigma}(t)=\frac{\left(1+t^{u}\right)\left(1+t^{u}\left(1+\sum_{\alpha \in \nabla_{s}} t^{<\alpha, \mu>}\right)+t^{2 u}\right)}{\prod_{\alpha \in \nabla_{l}}\left(1-t^{<\alpha, \mu>+u}\right)} .
$$

Since $\lambda_{2}$ is a long root, the images of $\lambda_{2}$ under the action of the Weyl group are exactly the long roots. In this form we just have positive coefficients in our numerator. The fully multiplied Hilbert series can be obtained by multiplying and diving the last expression with

$$
\left(1-t^{u}\right)^{2} \prod_{\alpha \in \nabla_{s}}\left(1-t^{<\alpha, \mu>+u}\right) .
$$

So we get the expression (4.5). The sum of the weights on $w \mathbb{P} V_{\lambda_{2}}$ is $14 u$, therefore the canonical bundle is $K_{w \Sigma}=\mathcal{O}(-3 u)$.

The Hilbert series of the straight flag variety $\Sigma \hookrightarrow \mathbb{P}^{13}$ can be computed to be

$$
P_{\Sigma}(t)=\frac{1-28 t^{2}+105 t^{3}-\cdots+105 t^{8}-28 t^{9}-t^{11}}{(1-t)^{14}} .
$$

The image is defined by 28 quadratic equations, listed in Appendix A.

\subsection{Examples}

4.7 Example The threefold linear section

$$
V=\Sigma \cap H_{1} \cap H_{2} \subset \mathbb{P}^{11}
$$

is a Fano threefold of genus 10, anti-canonically polarised by $-K_{V}$ with $\left(-K_{V}\right)^{3}=18$. This variety was constructed by Mukai using the vector bundle method in [10].

In fact, we failed to find any other $\mathbb{Q}$-Fano which is a quasilinear section of a codimension 8 weighted $G_{2}$ flag variety or a cone over such. A list of 487 possible codimension 8 $\mathbb{Q}$-Fano 3-folds with terminal singularities is available on Gavin Brown's graded ring data base page [4]. We checked that none of them matches the format above.

4.8 Example We construct a family of Calabi-Yau threefolds as quasi-linear sections of the weighted $G_{2}$ flag variety $w \Sigma \hookrightarrow \mathbb{P} V_{\lambda_{2}}$.

- Input data: $\mu=2 \beta_{1}-3 \beta_{2}, u=4$. 
- Weighted flag variety: $w \Sigma \subset \mathbb{P}^{13}\left[1^{2}, 2,3^{2}, 4^{4}, 5^{2}, 6,7^{2}\right]$, with weights assigned to the variables $x_{i}$ in the equations of Appendix A:

$\begin{array}{ccccccccccccccc}\text { Variable } & x_{1} & x_{2} & x_{3} & x_{4} & x_{5} & x_{6} & x_{7} & x_{8} & x_{9} & x_{10} & x_{11} & x_{12} & x_{13} & x_{14} \\ \text { Weight } & 6 & 1 & 3 & 5 & 7 & 4 & 2 & 7 & 5 & 3 & 1 & 4 & 4 & 4\end{array}$

- Canonical class: $K_{w \Sigma}=\mathcal{O}(-3 u)=\mathcal{O}(-12)$, since $w \Sigma$ is well-formed.

- Hilbert numerator: $1-t^{4}-2 t^{5}-4 t^{6}-2 t^{7}-t^{8}+\cdots+t^{44}$.

We take a 3 -fold quasilinear section

$$
X=w \Sigma \cap\left\{x_{4}=f_{5}\right\} \cap\left\{x_{8}=f_{7}\right\} \subset \mathbb{P}^{11}\left[1^{2}, 2,3^{2}, 4^{4}, 5,6,7\right],
$$

where $f_{5}$ and $f_{7}$ are general homogeneous forms of degree 5 , and 7 respectively in the remaining variables, so that

$$
K_{X}=\mathcal{O}(-12+(7+5))=0
$$

One can verify that $X$ is well-formed as it does not contain any codimension 9 singular stratum. We now check the singularities of $X$ arising from the weights of $w \mathbb{P}$.

$1 / 7$ singularities: This stratum is a single point in $w \mathbb{P}$ and it is readily seen that $X$ contains this singular point. We use the implicit function theorem to work out local variables near this point. We can eliminate the variables $x_{11}, x_{10}, x_{12}, x_{13}, x_{7}, x_{2}, x_{9}$, and $x_{3}$ from equations (A.1,6,9,11,12,19,26,28) of Appendix A, respectively. Therefore we get $x_{1}, x_{6}$ and $x_{14}$ as local variables near this point on an orbifold cover, the group $\mu_{7}$ acting by

$$
\varepsilon: x_{1}, x_{6}, x_{14} \mapsto \varepsilon^{6} x_{1}, \varepsilon^{4} x_{4}, \varepsilon^{4} x_{14}
$$

So this is an isolated singular point in $X$ of type $\frac{1}{7}(6,4,4)$.

$1 / 6$ singularities: This stratum is defined by

$$
X \cap\left\{x_{2}=x_{3}=x_{5}=x_{6}=x_{7}=x_{9}=x_{10}=x_{11}=x_{12}=x_{13}=x_{14}=0\right\} .
$$

This gives $x_{1}^{2}=0$, which has only a trivial solution. So $X$ does not contain this singular point.

1/5 singularities: We have only one variable $x_{9}$ of weight 5 and some pure power of $x_{9}$ appears in the defining equations of $X$, so it does not contain $1 / 5$ singular points.

1/4 singularities: $X$ restricted to this stratum is defined by

$$
\left\{x_{1}=x_{2}=x_{3}=x_{5}=x_{7}=x_{9}=x_{10}=x_{11}=0\right\} .
$$

We get a rational curve

$$
C=\left\{x_{6} x_{12}+x_{14}^{2}=0\right\} \subset \mathbb{P}^{2}\left[x_{6}, x_{12}, x_{14}\right]
$$


of singularities. Standard calculations show that it is a curve of type $\frac{1}{4}(1,3)$.

$1 / 3$ singularities: On this stratum, the equations again have only the trivial solution, so $X$ does not contain $1 / 3$ singularities.

$1 / 2$ singularities: On this stratum $X$ does not contain any new singularities, apart from the curve $C$ above.

Quasi-smoothness: We have already shown that on singular strata $X$ is locally a threefold by finding the local variables by using the implicit function theorem. One can show by similar calculations that on the rest of the strata, $X$ is locally a smooth threefold. Therefore $(X, D)$ is quasi-smooth.

In summary, $(X, D)$ belongs to a family of a polarised Calabi-Yau 3-folds with an isolated singular point of type $\frac{1}{7}(6,4,4)$ and a rational curve of singularities $C$ of type $\frac{1}{4}(1,3)$. By using the Riemann-Roch formula for Calabi-Yau threefolds ([5, Section 3]), we can compute the rest of the invariants of this family. We get the following data.

$$
\begin{aligned}
& \text { - } D^{3}=\frac{45}{56}, \quad D \cdot c_{2}(X)=\frac{150}{7} . \\
& \text { - }\left.\operatorname{deg} D\right|_{C}=\frac{1}{2}, N_{C}=-4 \text { and } \tau_{C}=1 .
\end{aligned}
$$

One can recover the Hilbert series of this variety following [5], by using these singularities as input data.

\section{$5 \quad$ Weighted $\operatorname{Gr}(2,6)$ varieties}

\subsection{The weighted flag variety}

We take $G$ to be the reductive Lie group of type $\operatorname{GL}(6, \mathbb{C})$ with maximal torus $T$. In the weight lattice $\Lambda_{W}=<e_{1}, e_{2}, e_{3}, e_{4}, e_{5}, e_{6}>$, the simple roots are $\left\{\alpha_{i}=e_{i}-e_{i+1}\right\}$, for $1 \leq i \leq 5$. The Weyl group of $G$ is $S_{6}$ of order 720 . The Weyl vector can be taken to be

$$
\rho=5 e_{1}+4 e_{2}+3 e_{3}+2 e_{4}+e_{5} .
$$

Consider the irreducible $G$-representation $V_{\lambda}$, with $\lambda=e_{1}+e_{2}$. Then $V_{\lambda}$ is 15 -dimensional, and all of the weights appear with multiplicity one. The highest weight orbit space $\Sigma=G / P_{\lambda} \subset \mathbb{P} V_{\lambda}=\mathbb{P}^{14}$ is eight dimensional. This flag variety can be identified with the Grassmannian of 2-planes in a 6 -dimensional vector space, a codimension 6 variety

$$
\Sigma^{8}=\operatorname{Gr}(2,6) \hookrightarrow \mathbb{P} V_{\lambda}=\mathbb{P}^{14} .
$$

Let $\left\{f_{i}, 1 \leq i \leq 6\right\}$ be the dual basis of the dual lattice $\Lambda_{W}^{*}$. We choose

$$
\mu=\sum_{i=1}^{6} a_{i} f_{i} \in \Lambda_{W}^{*},
$$


and $u \in \mathbb{Z}$, to get the weighted version of $\operatorname{Gr}(2,6)$,

$$
w \Sigma(\mu, u)=w \operatorname{Gr}(2,6)_{(\mu, u)} \hookrightarrow w \mathbb{P}^{14} .
$$

The set of weights on our projective space is $\left\{\left\langle w_{i}, \mu\right\rangle+u\right\}$, where $w_{i}$ are weights appearing in the $G$-representation $V_{\lambda}$. As a convention we will write an element of dual lattice as row vector, i.e. $\mu=\left(a_{1}, a_{2}, \cdots, a_{6}\right)$.

We expand the formula (3.2) for the given values of $\lambda, \mu$ to get the following formula for the Hilbert series of $w \operatorname{Gr}(2,6)$.

$$
P_{w \operatorname{Gr}(2,6)}(t)=\frac{1-Q_{1}(t) t^{2 u}+Q_{2}(t) t^{3 u}-Q_{3}(t) t^{4 u}-Q_{4}(t) t^{5 u}+Q_{5}(t) t^{6 u}-Q_{6}(t) t^{7 u}+t^{3 s+9 u}}{\prod_{1 \leq i<j \leq 6}\left(1-t^{a_{i}+a_{j}+u}\right)} .
$$

Here

$$
\begin{gathered}
Q_{1}(t)=\sum_{1 \leq i<j \leq 6} t^{s-\left(a_{i}+a_{j}\right)}, \quad Q_{2}(t)=\sum_{1 \leq(i, j) \leq 6} t^{s+\left(a_{i}-a_{j}\right)}-t^{s}, \\
Q_{3}(t)=\sum_{1 \leq i \leq j \leq 6} t^{s+\left(a_{i}+a_{j}\right)}, \quad Q_{4}(t)=\sum_{1 \leq i \leq j \leq 6} t^{2 s-\left(a_{i}+a_{j}\right)}, \\
Q_{5}(t)=\sum_{1 \leq(i, j) \leq 6} t^{2 s+\left(a_{i}-a_{j}\right)}-t^{2 s}, \quad Q_{6}(t)=\sum_{1 \leq i \leq j \leq 6} t^{2 s+\left(a_{i}+a_{j}\right)} .
\end{gathered}
$$

In particular, if $w \operatorname{Gr}(2,6) \hookrightarrow \mathbb{P}^{14}\left[<w_{i}, \mu>+u\right]$ is well-formed, then its canonical bundle is $K_{w \operatorname{Gr}(2,6)}=\mathcal{O}(-2 s-6 u)$, with $s=\sum_{i=1}^{6} a_{i}$.

The defining equations for $\operatorname{Gr}(2,6) \subset \mathbb{P}^{14}$ are well known to be the $4 \times 4$ Pfaffians, obtained by deleting two rows and the corresponding columns of the $6 \times 6$ skew symmetric matrix

$$
A=\left[\begin{array}{cccccc}
0 & x_{1} & x_{2} & x_{3} & x_{4} & x_{5} \\
& 0 & x_{6} & x_{7} & x_{8} & x_{9} \\
& & 0 & x_{10} & x_{11} & x_{12} \\
& & & 0 & x_{13} & x_{14} \\
& & & & 0 & x_{15} \\
& & & & & 0
\end{array}\right] .
$$

\subsection{Examples}

5.3 Example For $u=1$ and $\mu=(\underline{0})$, we get the Hilbert series of $\operatorname{Gr}(2,6)$.

$$
P_{\mathrm{Gr}(2,6)}(t)=\frac{1-15 t^{2}+35 t^{3}-21 t^{4}-21 t^{5}+35 t^{6}-15 t^{7}+t^{9}}{(1-t)^{15}} .
$$

We have $K_{\mathrm{Gr}(2,6)}=\mathcal{O}(-6)$. The three fold hyperplane section

$$
V=\Sigma \cap H_{1} \cap H_{2} \cap H_{3} \cap H_{4} \cap H_{5} \subset \mathbb{P}^{9},
$$

is a Fano 3 -fold with $\left(-K_{V}\right)^{3}=14$ and genus $g=8$. This variety appears in [10].

We searched for more examples of $\mathbb{Q}$-Fano threefolds as quasi-linear sections of weighted $\operatorname{Gr}(2,6)$, but as before, didn't succeed. 
5.4 Example Consider the following data.

- Input: $\mu=(2,1,0,0,-1,-2), u=4$

- Variety and Weights: $w \operatorname{Gr}(2,6) \subset \mathbb{P}^{14}\left[1,2^{2}, 3^{3}, 4^{3}, 5^{3}, 6^{2}, 7\right]$

- Canonical class: $K_{w \operatorname{Gr}(2,6)}=\mathcal{O}(-24)$

- Hilbert Numerator: $1-t^{5}-2 t^{6}-3 t^{7}-2 t^{8}-t^{9}+\cdots-3 t^{29}-2 t^{30}-t^{31}+t^{36}$.

Consider the threefold quasilinear section

$$
X=w \operatorname{Gr}(2,6) \cap(6) \cap(5)^{2} \cap(4)^{2} \subset \mathbb{P}^{9}\left[1,2^{2}, 3^{3}, 4,5,6,7\right],
$$

then

$$
K_{X}=\mathcal{O}(24-(6+2 \times 5+2 \times 4) \sim 0 .
$$

As in Example 4.8, it is easy to check that $(X, D)$ is well-formed and quasi-smooth. The singularities of $(X, D)$ can also be worked out as before. The conclusion is that $(X, D)$ is a Calabi-Yau threefold with an isolated singular point $\frac{1}{7}(6,5,3)$ and a dissident singular point $\frac{1}{6}(1,2,3)$ which lies on the intersection of two rational curves of singularities $C$ and $E$ of types $\frac{1}{3}(1,2)$ and $\frac{1}{2}(1,1)$ respectively. The rest of data of our variety and its singularities is as follows.

- $D^{3}=\frac{11}{21}, D \cdot c_{2}(X)=16$.

- $\left.\operatorname{deg} D\right|_{C}=\frac{1}{2}, N_{C}=3$ and $\tau_{C}=2$.

- $\left.\operatorname{deg} D\right|_{E}=\frac{2}{3}, N_{E}=1$ and $\tau_{E}=1$. 


\section{A Equations of the $G_{2}$ flag variety}

The codimension eight $G_{2}$ flag variety is defined by the following 28 quadrics.

$$
\begin{aligned}
& x_{14}^{2}+x_{13} x_{14}+\frac{1}{3} x_{13}^{2}+x_{6} x_{12}+\frac{1}{3} x_{4} x_{10}+x_{5} x_{11}+\frac{1}{3} x_{3} x_{9}+x_{2} x_{8}+\frac{1}{3} x_{1} x_{7} \\
& x_{14}^{2}+2 x_{13} x_{14}+\frac{8}{9} x_{13}^{2}+x_{6} x_{12}+3 x_{5} x_{11}-\frac{4}{9} x_{4} x_{10}-\frac{7}{9} x_{3} x_{9}-\frac{5}{9} x_{1} x_{7} \\
& 2 x_{13} x_{14}+\frac{5}{3} x_{13}^{2}+9 x_{5} x_{11}-\frac{5}{3} x_{4} x_{10}-\frac{7}{3} x_{3} x_{9}+3 x_{2} x_{8}-\frac{4}{3} x_{1} x_{7} \\
& x_{13}^{2}+9 x_{5} x_{11}-3 x_{4} x_{10}-3 x_{3} x_{9}+9 x_{2} x_{8}-2 x_{1} x_{7} \\
& x_{11} x_{14}+\frac{1}{3} x_{11} x_{13}+\frac{1}{9} x_{7} x_{10}+x_{2} x_{12} \\
& x_{5} x_{10}-\frac{2}{3} x_{4} x_{9}-3 x_{3} x_{8}+2 x_{1} x_{14}+\frac{1}{3} x_{1} x_{13} \\
& x_{10} x_{14}+\frac{4}{9} x_{10} x_{13}+\frac{2}{9} x_{7} x_{9}-x_{3} x_{12}+\frac{1}{3} x_{1} x_{11} \\
& x_{9} x_{14}+x_{9} x_{13}-x_{7} x_{8}+x_{4} x_{12}+\frac{2}{3} x_{1} x_{10} \\
& x_{8} x_{14}+\frac{2}{3} x_{8} x_{13}-x_{5} x_{12}+\frac{1}{9} x_{1} x_{9} \\
& 2 x_{7} x_{14}+\frac{5}{3} x_{7} x_{13}-3 x_{4} x_{11}-\frac{2}{3} x_{3} x_{10}+x_{2} x_{9} \\
& x_{6} x_{8}+x_{5} x_{14}+\frac{1}{3} x_{5} x_{13}+\frac{1}{9} x_{1} x_{4} \\
& 3 x_{5} x_{7}-x_{6} x_{9}+x_{4} x_{14}+\frac{4}{3} x_{4} x_{13}-\frac{2}{3} x_{1} x_{3} \\
& x_{6} x_{10}-\frac{2}{3} x_{4} x_{7}+x_{3} x_{14}-\frac{1}{3} x_{3} x_{13}+3 x_{1} x_{2} \\
& -x_{6} x_{11}+\frac{1}{9} x_{3} x_{7}+x_{2} x_{14}+\frac{2}{3} x_{2} x_{13} \\
& x_{12} x_{13}-\frac{1}{3} x_{9} x_{10}+3 x_{8} x_{11} \\
& x_{10} x_{13}-x_{7} x_{9}+3 x_{1} x_{11} \\
& x_{9} x_{13}-3 x_{7} x_{8}+x_{1} x_{10} \\
& x_{7} x_{13}-3 x_{4} x_{11}+3 x_{2} x_{9} \\
& x_{6} x_{13}-\frac{1}{3} x_{3} x_{4}+3 x_{2} x_{5} \\
& 3 x_{5} x_{7}+x_{4} x_{13}-x_{1} x_{3} \\
& x_{4} x_{7}+x_{3} x_{13}-3 x_{1} x_{2} \\
& x_{1} x_{13}-3 x_{5} x_{10}+3 x_{3} x_{8} \\
& x_{9} x_{11}-\frac{1}{3} x_{10}^{2}+x_{7} x_{12} \\
& \frac{1}{3} x_{9}^{2}-x_{8} x_{10}+x_{1} x_{12} \\
& \frac{1}{3} x_{7}^{2}+x_{3} x_{11}+x_{2} x_{10} \\
& x_{5} x_{9}+x_{4} x_{8}+\frac{1}{3} x_{1}^{2} \\
& x_{6} x_{7}+\frac{1}{3} x_{3}^{2}-x_{2} x_{4} \\
& x_{1} x_{6}-\frac{1}{3} x_{4}^{2}+x_{3} x_{5}
\end{aligned}
$$

This list was obtained using the algorithm described above in Section 3.5, with the help of the GAP4 code given below, kindly provided to us by Willem De Graaf. With obvious modifications, this code in principle computes the equations of any flag variety. 


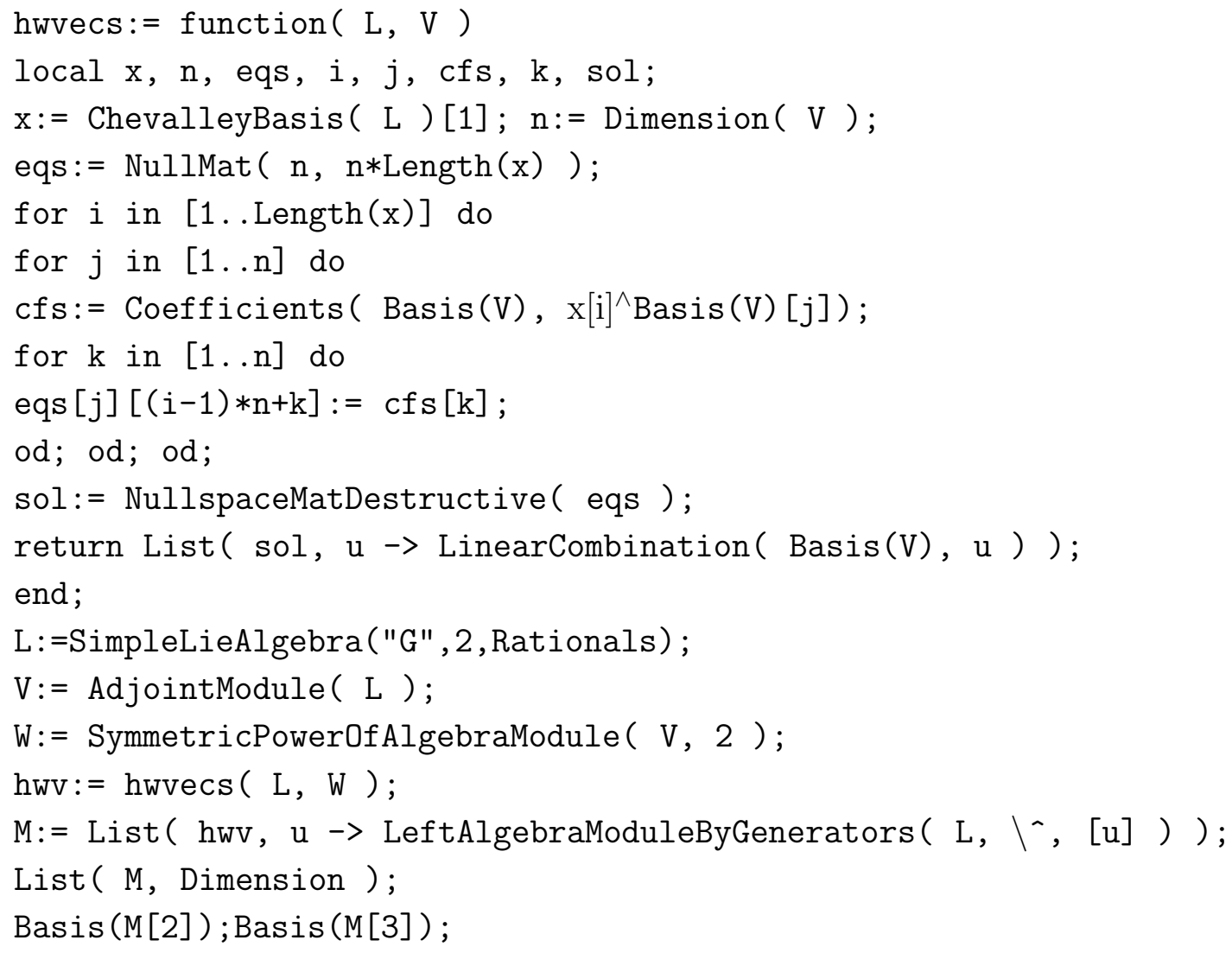

\section{References}

[1] S. Altmok, Graded rings corresponding to the polarised $K 3$ surfaces and $\mathbb{Q}$-Fano 3-folds, PhD. thesis, University of Warwick, 1998.

[2] S. Altınok, G. Brown, and M. Reid, Fano 3-folds, K3 surfaces and graded rings, Topology and geometry: commemorating SISTAG (eds. Berrick, Leung, Xu), Contemp. Math., 314, 25-53, AMS, 2002.

[3] Atiyah, M. F. and Macdonald, I. G., Introduction to commutative algebra, AddisonWesley, 1969.

[4] G. Brown, Graded ring database, http://grdb.lboro.ac.uk/.

[5] A. Buckley and B. Szendrői, Orbifold Riemann-Roch for threefolds with an application to Calabi-Yau geometry, J. Algebraic Geom., 14, 601-622, 2005.

[6] A. Corti and M. Reid, Weighted Grassmannians, Algebraic geometry (eds. Beltrametti, Catanese, Ciliberto, Lanteri, Pedrini), 141-163, de Gruyter, 2002.

[7] W. Fulton and J. Harris, Representation Theory, A first course, GTM 129, Springer, 1991. 
[8] W. A. de Graaf, Constructing representations of split semisimple Lie algebras, Effective methods in algebraic geometry (Bath, 2000), J. Pure Appl. Algebra, 164, 87-107, 2001.

[9] A.L. Gorodentsev, A.S. Khoroshkin, and A. N. Rudakov, On syzygies of highest weight orbits, Moscow Seminar on Mathematical Physics. II (eds. Arnold, Gindikin, Maslov), Amer. Math. Soc. Transl. Ser. 2, 221, 79-120, AMS, 2007.

[10] S. Mukai, Curves, $K 3$ surfaces and Fano 3-folds of genus $\leq 10$, in: Algebraic geometry and commutative algebra, Vol. I (eds. Hijikata, Hironaka, Maruyama, Matsumura, Miyanishi, Oda, Ueno), 357-377, Kinokuniya, 1988.

[11] M. I. Qureshi, D.Phil. thesis, University of Oxford, in preparation.

[12] The GAP group: GAP - Groups, Algorithms, and Programming, Version 4.4.12, http://www.gap-system.org, 2008.

Mathematical Institute, University of Oxford.

24-29 St Giles', Oxford, OX1 3LB, United Kingdom.

qureshi@maths.ox.ac.uk

szendroi@maths.ox.ac.uk 